\title{
THE ALGEBRA OF DECOMPOSABLE OPERATORS IN DIRECT INTEGRALS OF NOT NECESSARILY SEPARABLE HILBERT SPACES
}

\author{
REINHARD SCHAFLITZEL
}

(Communicated by Paul S. Muhly)

\begin{abstract}
Considering direct integrals of not necessarily separable Hilbert spaces we examine the question whether the algebra of decomposable operators is the commutant of the algebra of diagonalizable operators. Using the continuum-hypothesis we prove this relation, if the set of square integrable vector fields is generated by a subset $\Gamma_{0}$ such that $\left|\Gamma_{0}\right| \leq|\mathbf{R}|$. For the general case, a counterexample is given.
\end{abstract}

In a direct integral of separable Hilbert spaces (for example see [1]) the relation $\mathscr{Z}=\mathscr{D}^{\prime}$ holds, where $\mathscr{Z}$ is the set of decomposable operators and $\mathscr{D}^{\prime}$ the commutant of the algebra $\mathscr{D}$ of diagonalizable operators. The same is also satisfied if a constant field of nonseparable Hilbert spaces is considered (for example see $[2 ; 5 ; 4$, Theorem IV.7.10]). In this paper we are concerned with the question whether the same is correct if we consider the more general concept of direct integrals of W. Wils (see [6]) in which nonseparable Hilbert spaces and nonconstant fields are permitted. Using the continuum-hypothesis, we can answer this question in the affirmative if the set of square integrable vector fields is generated by a subset of the cardinality of the continuum. Moreover, an example for $\mathscr{Z} \neq \mathscr{D}^{\prime}$ will be given, for which we need the generalized continuum-hypothesis.

Similar problems as in this article are treated in [2], [3], [5]. (The proof of Proposition 3.1 in [5] contains an error; it is not known whether the proposition is correct.) In [3, Théorème 4.5] a sufficient condition for $\mathscr{Z}=\mathscr{D}^{\prime}$ is given; but in many concrete situations it is difficult or impossible to verify the assumptions there.

Let us recall the definition of direct integrals introduced by $\mathrm{W}$. Wils.

1. Definition. Let $(\mathrm{Z}, \mu)$ be a measure space and $H(\zeta)$ be a complex Hilbert space for $\zeta \in Z$. A linear subspace $\Gamma$ of $\prod_{\zeta \in Z} H(\zeta)$ is called a set of $\mu$-square

Received by the editors July 24,1989 .

1980 Mathematics Subject Classification (1985 Revision). Primary 46L45.

Key words and phrases. Direct integrals, algebra of decomposable operators, algebra of diagonalizable operators, continuum-hypothesis. 
integrable vector fields, iff

(1) $\zeta \in Z \longrightarrow\|\eta(\zeta)\|^{2}$ is $\mu$-integrable for every $\eta=(\eta(\zeta))_{\zeta \in Z} \in \Gamma$.

(2) If for $\eta \in \prod_{\zeta \in Z} H(\zeta)$ there is a $\eta^{\prime} \in \Gamma$ such that $\eta(\zeta)=\eta^{\prime}(\zeta)$ for almost every $\zeta$, then $\eta \in \Gamma$.

(3) If $\eta \in \Gamma$ and $h \in L^{\infty}(Z, \mu)$, then $h \cdot \eta=(h(\zeta) \eta(\zeta))_{\zeta \in Z} \in \Gamma$.

(4) $\Gamma$ with respect to the seminorm $\|\eta\|_{2}=\left(\int_{Z}\|\eta(\zeta)\|^{2} d \mu(\zeta)\right)^{1 / 2}$ is complete.

The corresponding Hilbert space, called the direct integral of the Hilbert spaces $H(\zeta)$, is denoted by

$$
H=\int_{Z}^{\Gamma} H(\zeta) d \mu(\zeta)
$$

2. Lemma. If $\Gamma_{0}$ is a subset of $\prod_{\zeta \in Z} H(\zeta)$ such that $\zeta \in Z \longmapsto<\eta(\zeta), \eta^{\prime}(\zeta)>$ is $\mu$-integrable for $\eta, \eta^{\prime} \in \Gamma_{0}$, there is a unique smallest subspace $\Gamma$ such that $\Gamma_{0} \subseteq \Gamma$ and $\Gamma$ satisfies (1)-(4).

In this situation, we say $\Gamma$ is generated by $\Gamma_{0}$.

Proof. Let $\widetilde{\Gamma}_{0}$ be the vector space generated by $\Gamma_{0}$. It follows that $\zeta \in Z \longmapsto$ $\|\eta(\zeta)\|^{2}$ is $\mu$-integrable for every $\eta \in \widetilde{\Gamma}_{0}$. Now, from [6, Corollary 2.3] we get the assertion.

An operator $T \in \mathscr{L}(H)$ is called decomposable if there is a field $(T(\zeta))_{\zeta \in Z} \subseteq$ $\prod_{\zeta \in Z} \mathscr{L}(H(\zeta))$ of operators such that $\sup _{\zeta \in Z}\|T(\zeta)\|<\infty, \quad(T(\zeta) \eta(\zeta))_{\zeta \in Z} \in$ $\Gamma$ for $\eta \in \Gamma$ and

$$
T \int_{Z}^{\Gamma} \eta(\zeta) d \mu(\zeta)=\int_{Z}^{\Gamma} T(\zeta) \eta(\zeta) d \mu(\zeta)
$$

holds for $\eta \in \Gamma$. We write $T=\int_{Z}^{\Gamma} T(\zeta) d \mu(\zeta)$.

Let $\mathscr{Z}$ be the set of decomposable operators. $\mathscr{Z}$ is an algebra, but it is not known whether $T \in \mathscr{Z}$ yields $T^{*} \in \mathscr{Z}$.

$\mathscr{D} \stackrel{\text { def }}{=}\left\{\int_{Z}^{\Gamma} h(\zeta) \mathbf{1} d \mu(\zeta): h \in L^{\infty}(Z, \mu)\right\}$ is a von Neumann algebra called the algebra of diagonalizable operators. Obviously, we have $\mathscr{Z} \subseteq \mathscr{D}^{\prime}$.

We call a subset $\Gamma_{0}$ of $\prod_{\zeta \in Z} H(\zeta)$ almost pointwise orthogonal, if $\eta(\zeta) \perp$ $\eta^{\prime}(\zeta)$ holds almost everywhere for all fixed distinct elements $\eta, \eta^{\prime}$ of $\Gamma_{0}$. (The negligible set may depend on $\eta$ and $\eta^{\prime}$.) We call $\Gamma_{0}$ pointwise orthogonal, if $\eta(\zeta) \perp \eta^{\prime}(\zeta)$ holds for every $\zeta \in Z$ and for all distinct elements $\eta, \eta^{\prime}$ of $\Gamma_{0}$.

3. Proposition (see $[2, \S 6]$ ). If $\Gamma$ is generated by a set $\Gamma_{0}$ of pointwise orthogonal vector fields, then $\mathscr{Z}=\mathscr{D}^{\prime}$ is satisfied.

Now let us formulate our main result:

4. Theorem. If $\Gamma$ is generated by a set $\widetilde{\Gamma}$ of vector fields such that $|\widetilde{\Gamma}| \leq \aleph_{1}$, then $\mathscr{Z}=\mathscr{D}^{\prime}$ holds. 
Immediately we get the following

5. Corollary. Let us assume the continuum-hypothesis. Moreover, let us suppose that the dimension of $H$ (as a Hilbert space) is not greater than the cardinality c of the continuum or, more generally, that $\Gamma$ is generated by a set $\widetilde{\Gamma}$ of vector fields such that $|\widetilde{\Gamma}| \leq \mathbf{c}$. Then $\mathscr{Z}=\mathscr{D}^{\prime}$ holds.

Proof of theorem. Using Zorn's lemma we easily see that there is a subset $\Gamma_{0}$ of $\Gamma$ with $\left|\Gamma_{0}\right| \leq \aleph_{1}$ such that $H$ is the direct orthogonal sum of the spaces $L_{\eta}, \eta \in \Gamma_{0}$, where $L_{\eta}$ denotes the closed subspace of $H$ generated by the vector fields $\int_{Z}^{\Gamma} h(\zeta) \eta(\zeta) d \mu(\zeta) \quad\left(h \in L^{\infty}(Z, \mu)\right)$.

$\Gamma_{0}$ is almost pointwise orthogonal: Let $\eta$ and $\eta^{\prime}$ be distinct elements of $\Gamma_{0} ; h \eta \in L_{\eta}$ holds for $h(\zeta)=<\eta^{\prime}(\zeta), \eta(\zeta)>$, and so the orthogonality of $L_{\eta}$ and $L_{\eta^{\prime}}$ implies that

$$
\zeta \in Z \longmapsto<h(\zeta) \eta(\zeta), \eta^{\prime}(\zeta)>=\left|<\eta(\zeta), \eta^{\prime}(\zeta)>\right|^{2}
$$

is equal to 0 almost everywhere.

Now we want to find a map $f: \Gamma_{0} \longrightarrow \prod_{\zeta \in Z} H(\zeta)$ such that $\eta(\zeta)=f(\eta)(\zeta)$ almost everywhere for fixed $\eta \in \Gamma$ and such that $f\left(\Gamma_{0}\right)$ is pointwise orthogonal. Then from Proposition 3 we will get the assertion.

In the case $\left|\Gamma_{0}\right| \leq \aleph_{0}$ it is easy to find a suitable map $f$. Let us assume $\left|\Gamma_{0}\right|=\aleph_{1}$. For an ordinal number $\alpha$, let $P_{\alpha}:=\{\beta ; \beta$ ordinal number, $\beta<$ $\alpha\}$. Moreover, let $\Omega$ denote the smallest ordinal number such that $P_{\Omega}$ is uncountable. Then $\left|P_{\Omega}\right|=\aleph_{1}$. Let $\alpha \longmapsto \eta_{\alpha}$ be a bijection between $P_{\Omega}$ and $\Gamma_{0}$. For $\alpha \in P_{\Omega}$ let

$$
N_{\alpha} \stackrel{\text { def }}{=} \bigcup_{\beta \in P_{o}}\left\{\zeta \in Z ;<\eta_{\beta}(\zeta), \eta_{\alpha}(\zeta)>\neq 0\right\} .
$$

We define

$$
f\left(\eta_{\alpha}\right)(\zeta) \stackrel{\text { def }}{=} \begin{cases}\eta_{\alpha}(\zeta) & \text { for } \zeta \in Z \backslash N_{\alpha} \\ 0 & \text { for } \zeta \in N_{\alpha} .\end{cases}
$$

Since $\left\{\zeta \in Z ;<\eta_{\beta}(\zeta), \eta_{\alpha}(\zeta)>\neq 0\right\}$ is a negligible set for every $\beta \in P_{\alpha}$ and $P_{\alpha}$ is countable by definition of $P_{\Omega}, N_{\alpha}$ is a negligible set. Moreover, for $\beta<\alpha, f\left(\eta_{\alpha}\right)(\zeta) \perp f\left(\eta_{\beta}\right)(\zeta)$ is valid for every $\zeta \in Z$. In the cases $f\left(\eta_{\alpha}\right)(\zeta)=0$ or $f\left(\eta_{\beta}\right)(\zeta)=0$, the equation is trivial. Otherwise from $f\left(\eta_{\alpha}\right)(\zeta)=\eta_{\alpha}(\zeta)$, $f\left(\eta_{\beta}\right)(\zeta)=\eta_{\beta}(\zeta)$, and $\eta_{\beta}(\zeta) \perp \eta_{\alpha}(\zeta)$ (since $\left.\zeta \notin N_{\alpha}\right)$ we get the desired relation. So, $f$ satisfies all the required properties.

In order to construct a counterexample we need the following

6. Lemma. Let us assume the generalized continuum-hypothesis. Let $\mathbf{a} \geq \mathbf{c}$ be a cardinal number such that $\mathbf{c} \leq|Z| \leq \mathbf{a}, \operatorname{dim} H(\zeta) \leq \mathbf{a}$ for every $\zeta \in Z$, and $\mu(Z)=1$. Moreover, let us suppose that $\Gamma$ is generated by an almost pointwise orthogonal set $\Gamma_{0}$ which satisfies $\left|\Gamma_{0}\right|>\mathbf{a}$ and $\|\eta(\zeta)\|=1$ for $\zeta \in Z$ and $\eta \in \Gamma_{0}$. Then $\mathscr{Z} \nsubseteq \mathscr{D}^{\prime}$. 
Proof. For a Hilbert space $K$, having an orthonormal base of the cardinality d $>\aleph_{0}$, it is well known that the algebra $\mathscr{L}(K)$ has the cardinality $2^{\text {d }}$.

It follows that

$$
|\mathscr{Z}| \leq\left|\prod_{\zeta \in Z} \mathscr{L}(H(\zeta))\right| \leq\left(2^{\mathrm{a}}\right)^{|Z|}=2^{\mathrm{a} \cdot|Z|}=2^{\mathrm{a}} .
$$

Let $\Gamma_{0}=\left\{\eta_{\alpha} ; \alpha \in A\right\}$, where $A$ is a suitable index set; let us choose an orthonormal base $\left\{e_{\alpha} ; \alpha \in A\right\}$ of $\ell^{2}(A)$. Then

$$
\int_{Z}^{\Gamma} h(\zeta) \eta_{\alpha}(\zeta) d \mu(\zeta) \longmapsto h \otimes e_{\alpha}
$$

defines a unitary operator between $H=\int_{Z}^{\Gamma} H(\zeta) d \mu(\zeta)$ and $L^{2}(Z, \mu) \otimes \ell^{2}(A)$, which transforms $\mathscr{D}$ into $L^{\infty}(Z, \mu) \otimes \mathbf{C} 1$, where $L^{\infty}(Z, \mu)$ is interpreted as the multiplication algebra in $L^{2}(Z, \mu)$. Thus $\mathscr{D}^{\prime}$ is transformed into $L^{\infty}(Z, \mu) \otimes \mathscr{L}\left(\ell^{2}(A)\right)$, and hence we get

$$
\left|\mathscr{D}^{\prime}\right| \geq\left|\mathscr{L}\left(\ell^{2}(A)\right)\right|=2^{|A|}=2^{\left|\Gamma_{0}\right|}>2^{\mathrm{a}},
$$

where in the last inequality $\left|\Gamma_{0}\right|>\mathbf{a}$ and the generalized continuum-hypothesis have been used. From $(*)$ we see that $\mathscr{Z}=\mathscr{D}^{\prime}$ is impossible.

7. Example. We again assume the generalized continuum-hypothesis. Let $Z=$ $[0,1], \mu$ the Lebesgue measure of $Z$, and $H(\zeta)=\ell^{2}(\mathbf{R})$ for $\zeta \in Z$. Let

$$
\begin{aligned}
\mathscr{O} \stackrel{\text { def }}{=}\left\{\tilde{\Gamma} \subseteq \prod_{\zeta \in Z} H(\zeta) ; \bar{\Gamma}\right. \text { almost pointwise orthogonal, } \\
\\
\|\eta(\zeta)\|=1 \text { for } \zeta \in Z \text { and } \eta \in \bar{\Gamma}\} .
\end{aligned}
$$

From Zorn's lemma it follows that $\mathscr{O}$ has maximal elements with respect to inclusion. Let $\Gamma_{0}$ be a maximal element. We show that the assumption $\left|\Gamma_{0}\right| \leq \mathrm{c}$ yields a contradiction. It is easy to see that $\left|\Gamma_{0}\right| \leq \aleph_{0}$ is impossible. In the case $\left|\Gamma_{0}\right|=\aleph_{1}=\mathbf{c}$, let $\alpha \longmapsto \eta_{\alpha}$ and $\alpha \longmapsto \zeta_{\alpha}$ be bijections from $P_{\Omega}$ (see the proof of the theorem, $\left|P_{\Omega}\right|=\aleph_{1}=\mathbf{c}$ ) to $\Gamma_{0}$ and $Z$, respectively. For $\alpha \in P_{\Omega}$ let $K\left(\zeta_{\alpha}\right)$ be the closed subspace of $H\left(\zeta_{\alpha}\right)$ generated by $\eta_{\beta}\left(\zeta_{\alpha}\right), \beta \in P_{\alpha}$. Since $P_{\alpha}$ is countable, $K\left(\zeta_{\alpha}\right)$ is separable, and there exists a vector $\xi\left(\zeta_{\alpha}\right) \in H\left(\zeta_{\alpha}\right)$ such that $\left\|\xi\left(\zeta_{\alpha}\right)\right\|=1$ and $\xi\left(\zeta_{\alpha}\right)$ is orthogonal to $K\left(\zeta_{\alpha}\right)$. For $\beta \in P_{\Omega}$ the relation $\eta_{\beta}\left(\zeta_{\alpha}\right) \perp \xi\left(\zeta_{\alpha}\right)$ is satisfied for $\alpha>\beta$; since $\left\{\alpha \in P_{\Omega} ; \alpha \leq \beta\right\}$ is countable, $\eta_{\beta}(\zeta) \perp \xi(\zeta)$ is valid for almost every $\zeta$. It follows that $\Gamma_{0} \cup\left\{\xi=(\xi(\zeta))_{\zeta \in Z}\right\}$ belongs to $\mathscr{O}$. This is a contradiction.

We have established $\left|\Gamma_{0}\right|>\mathbf{c}$. Let $\Gamma_{1}$ be a subset of $\Gamma_{0}$ such that $\left|\Gamma_{1}\right|>\mathbf{c}$. For $\eta, \eta^{\prime} \in \Gamma_{1}$ the function $\zeta \in Z \longmapsto<\eta(\zeta), \eta^{\prime}(\zeta)>$ is $\mu$-integrable; in fact, we see that it is equal to 0 or to 1 almost everywhere. From Lemma 2 we conclude that there is a unique smallest subspace $\Gamma$ such that $\Gamma_{1} \subseteq \Gamma$ and such that $\Gamma$ satisfies Definition 1. For $\Gamma$ we have $\mathscr{Z} \nsubseteq \mathscr{D}^{\prime}$ by Lemma 6 . 


\section{ACKNOWLEDGMENT}

It is a pleasure to thank Prof. R.W. Henrichs and Dr. D. Castrigiano for discussions and for their interest.

\section{REFERENCES}

1. J. Dixmier, von Neumann algebras, North-Holland, Amsterdam, New York, and Oxford, 1981.

2. E. T. Kehlet, Disintegration theory on a constant field of non-separable Hilbert spaces, Math. Scand. 43 (1978), 353-362.

3. O. Maréchal, Champs mesurables d'espaces hilbertiens, Bull. Sci. Math. 93 (1969), 113-143.

4. M. Takesaki, Theory of operator algebras I, Springer-Verlag, New York, Heidelberg, and Berlin, 1979.

5. J. Vesterstrom and W. Wils, Direct integrals of Hilbert spaces II, Math. Scand. 26 (1970), 89-102.

6. W. Wils, Direct integrals of Hilbert spaces I, Math. Scand. 26 (1970), 73-88.

InSTItUt Für MAThematiK der TeChNisChen Universität MÜNChen, ARCisstr. 21, D-8000 MÜNCHEN 2 